\title{
Numerical investigation of a small footprint plasmonic Bragg grating structure with a high extinction ratio
}

\author{
Muhammad Ali Butt ${ }^{1,2}$ \\ ${ }^{1}$ Department of Technical Cybernetics, Samara National Research University, 34 Moskovkoye Shosse, Samara \\ 443086, Russia \\ ${ }^{2}$ Institute of Microelectronics and Optoelectronics, Warsaw University of Technology, Koszykowa 75, 00-662 \\ Warszawa, Poland
}

Received August 19, 2020; accepted September 21, 2020; published September 30, 2020

\begin{abstract}
In this paper, miniaturized design of a plasmonic Bragg grating filter is investigated via the finite element method (FEM). The filter is based on a plasmonic metal-insulator-metal waveguide deposited on a quartz substrate. The corrugated Bragg grating designed for nearinfrared wavelength range is structured on both sides of the waveguide. The spectral characteristics of the filter are studied by varying the geometric parameters of the filter design. As a result, the maximum $E R$ and bandwidth of $36.2 \mathrm{~dB}$ and $173 \mathrm{~nm}$ are obtained at $\lambda_{\text {Bragg }}=976 \mathrm{~nm}$ with a filter footprint as small as $1.0 \times 8.75 \mu \mathrm{m}^{2}$, respectively. The $E R$ and bandwidth can be further improved by increasing the number of grating periods and the strength of the grating, respectively. Moreover, the Bragg grating structure is quite receptive to the refractive index of the medium. These features allow the employment of materials such as polymers in the metal-insulator-metal waveguide which can be externally tuned or it can be used for refractive index sensing applications. The sensitivity of the proposed Bragg grating structure can offer a sensitivity of $950 \mathrm{~nm} / \mathrm{RIU}$. We believe that the study presented in this paper provides a guideline for the realization of small footprint plasmonic Bragg grating structures which can be employed in filter and refractive index sensing applications.
\end{abstract}

Bragg gratings have been broadly used in photonic circuits incorporated on a silicon-on-insulator (SOI) platform to realize functionalities such as filter, coupler, medical diagnostics, spectroscopy, display colour generation and sensing, among others [1-3]. Bragg gratings based on SOI platform can provide these filtering characteristics on a low-cost platform but the main downside is the requirement of long grating structures to obtain high extinction ratio $(E R)$ [4-6]. It can be a major concern when filters with small footprints are preferred.

Surface plasmon polaritons (SPPs) are electromagnetic waves that propagate along the metal-insulator interface having an exponentially decaying field [7]. They offer the potential to develop highly integrated optical circuits due to their capability to overcome the diffraction limit of light. There are two main types of SPP based waveguide structures, namely: insulator-metal-insulator (IMI) and metal-insulator-metal (MIM) waveguides [7]. An MIM waveguide has the potential to squeeze the mode size down to a few tens of nanometer, which makes them an ideal candidate for nanoscale photonic devices. On the other hand, an IMI waveguide fails to do so. There are numerous plasmonic filter configurations that have been proposed such as ring resonator, Fabry-Perot resonator and Bragg grating, among others [8-10]. The Bragg grating based on the MIM structure can be realized by waveguide width modulation, waveguide core refractive index modulation and a combination of them [11]. Another significant application for the plasmonic Bragg grating is sensing, as the Bragg wavelength $\left(\lambda_{\text {Bragg }}\right)$ is highly receptive to external environments such as temperature, pressure and refractive index [12-13].

In this letter, we numerically investigate the effect of geometric parameters on the performance of the plasmonic Bragg grating structure. Here, we have concentrated on obtaining a high extinction ratio $(E R)$ of the Bragg grating filter, which is calculated as:

$$
E R=10 \times \log \frac{P_{\text {out }}}{P_{\text {in }}},
$$

where $P_{\text {out }}$ and $P_{\text {in }}$ are the output and input power, respectively. The proposed plasmonic Bragg grating filter design is shown in Fig. 1. The metal and insulator materials are defined as gold $(A u)$ and air, respectively. A metal-insulator-metal (MIM) waveguide with periodic corrugation is formed on both sides of the $A u$ layer resulting in a Bragg grating pattern. The frequencydependent complex relative permittivity of $A u$ is characterized by the Lorentz-Drude model. The width of the MIM waveguide is represented as $w$ which is fixed at $100 \mathrm{~nm}$. $L$ is the length of the segment where the waveguide width is $w$ whereas $s$ is the corrugated part while $d$ is the strength of corrugation. Therefore the period $(\Lambda)$ can be written as $\Lambda=L+s$. To simplify the study $s$ is fixed at $100 \mathrm{~nm}$. The number of grating period is expressed as $N$. The TM mode of the MIM waveguide is excited by a plane wave and the transmission spectrum is obtained by line integration of the output port. As $w<<$ $\lambda_{\text {incident }}$, thus, only the fundamental TM mode can exist. The transmission spectrum and E-field distributions are simulated using the 2D-finite element method (FEM) with a scattering boundary condition. The EM-wave frequency domain $(e m w)$ is selected as a physics interface and modal analysis is added to the study. In COMSOL simulations, the subdomains of the device design are divided into triangular mesh elements with an extra-fine mesh grid 
size for the MIM waveguide and a finer mesh grid for the air domain. This produces precise simulation results within the available computational resources. It is necessary to model a domain with open boundaries, that is such boundaries of the computational domain through which an EM wave can travel without reflection. An open geometry is estimated by employing scattering boundary conditions (SBC) at the outer edges of the FEM simulation window.

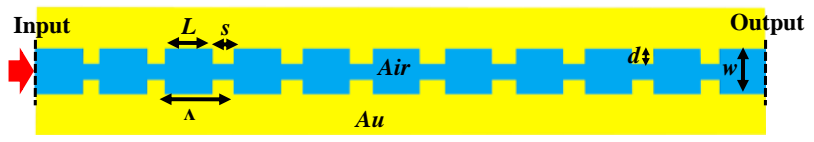

Fig. 1. 2D representation of a plasmonic Bragg grating filter.

Here, the spectral characteristics of the Bragg grating are studied by individually varying the geometric parameters of the structure. At first, the effect on $\lambda_{B r a g g}$ and $E R$ is examined by varying $\Lambda$ of the Bragg grating. To simplify the mechanism, $s$ is fixed at $100 \mathrm{~nm}$ whereas $L$ is varied between $200 \mathrm{~nm}$ and $300 \mathrm{~nm}$ with a step size of $25 \mathrm{~nm}$. The remaining parameters such as $d, w$ and $N$ are set at 20 $\mathrm{nm}, 100 \mathrm{~nm}$ and 15, respectively. From Fig. 2a, it can be observed that with increasing $L$ (or $\Lambda$ ), $\lambda_{\text {Bragg }}$ performs a redshift with a decrease in $E R$. At $L=200 \mathrm{~nm}, \lambda_{\text {Bragg }}=850$ $\mathrm{nm}$ having $E R=24.8 \mathrm{~dB}$ is obtained which shifts to $\lambda_{\text {Bragg }}=1114 \mathrm{~nm}$ with a slight decrease in $E R=19.73 \mathrm{~dB}$ at $L=300 \mathrm{~nm}$ as shown in Fig. 2a. To improve the $E R$, we can either increase $d$ or $N$. However, in the former case, the bandwidth can also increase. In the second stage, we analyzed the effect of $d$ on the $E R$ of the filter. The remaining parameters such as $L, s, w$ and $N$ are fixed at $250 \mathrm{~nm}, 100 \mathrm{~nm}, 100 \mathrm{~nm}$ and 15, respectively. From Fig. $2 \mathrm{~b}$, it can be seen that there is a noteworthy rise in the $E R$ of the filter along with the bandwidth as $d$ increases. The spectral characteristics of the filter are shown in Table 1.

Table 1. Spectral characteristics of the Bragg grating filter for different values of $d$.

\begin{tabular}{|c|c|c|c|}
\hline$d(\mathrm{~nm})$ & $\lambda_{\text {Bragg }}(\mathrm{nm})$ & $E R(d B)$ & Bandwidth $(\mathrm{nm})$ \\
\hline 10 & 918 & 10.34 & 86 \\
\hline 15 & 946 & 15.8 & 116 \\
\hline 20 & 982 & 21.9 & 157 \\
\hline 25 & 1030 & 28.74 & 218.8 \\
\hline 30 & 1094 & 36.47 & 327 \\
\hline
\end{tabular}

The number of periods $(N)$ has a vital impact on the $E R$ of the filter. We simulated plasmonic Bragg filter of $\Lambda=350$ $\mathrm{nm}$ and different values of $N$. The $E R$ is plotted for $N=5$, 10, 15, 20 and 25 as shown in Fig. 2 c. We can find an enhancement in $E R$ as $N$ increases with a slight change in the bandwidth of the filter. The $\lambda_{\text {Bragg }}, E R$, bandwidth and the footprint of the filter are presented in Table 2. This footprint is smaller than most of the previously reported filters [14-16].
Table 2: Spectral characteristics of the Bragg grating filter for different values of $N$.

\begin{tabular}{|c|c|c|c|c|}
\hline$N$ & $\lambda_{\text {Bragg }}(\mathrm{nm})$ & $E R(d B)$ & Bandwidth $(\mathrm{nm})$ & Footprint $\left(\mu \mathrm{m}^{2}\right)$ \\
\hline 5 & 1016 & 7.9 & 245 & $1 \times 1.75$ \\
\hline 10 & 988 & 14.8 & 194 & $1 \times 3.5$ \\
\hline 15 & 982 & 21.9 & 188 & $1 \times 5.25$ \\
\hline 20 & 978 & 29 & 179 & $1 \times 7$ \\
\hline 25 & 976 & 36.2 & 173 & $1 \times 8.75$ \\
\hline
\end{tabular}

It is worth noting that the $E R$ of the proposed Bragg grating filter can be further increased by increasing the value of $N$. To verify the idea, we have shown only limited values in this paper.
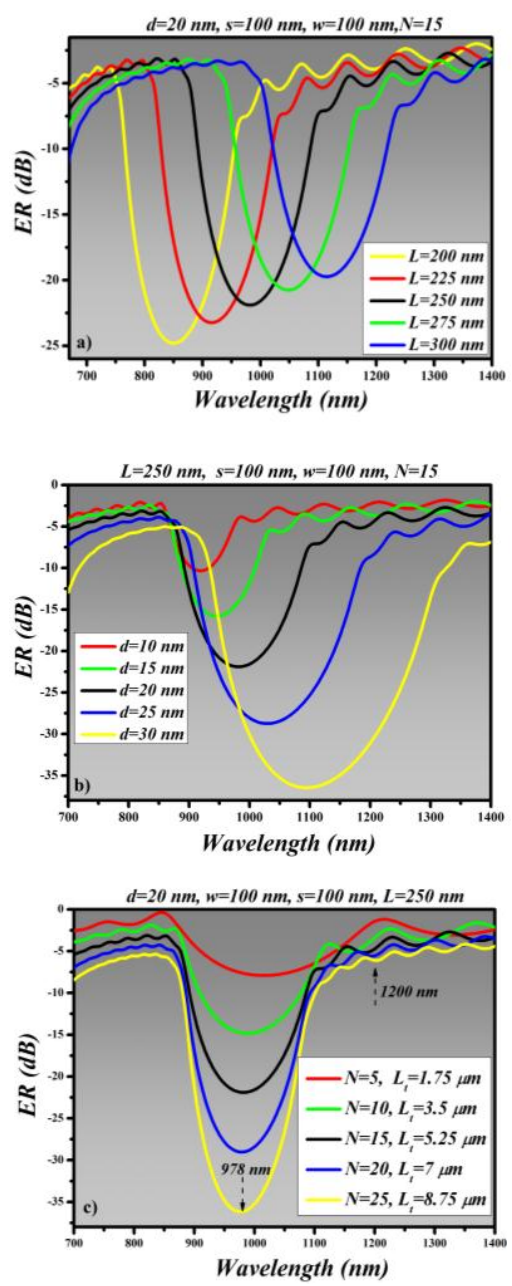

Fig.2. Extinction ratio dependent on, a) $L$, b) $d$ and, c) $N$. The remaining parameters of the grating structure is mentioned in the paper.

The E-field distribution of the propagating mode at $\lambda=$ $978 \mathrm{~nm}$ and $\lambda=1200 \mathrm{~nm}$ is plotted for $N=25$ as shown in Fig. 3. The remaining geometric parameters of the filter are the same as those used in Fig. $2 \mathrm{c}$. At $\lambda=978 \mathrm{~nm}$, the Bragg condition is satisfied, which results in a strong reflection of light $(E R=36.2 \mathrm{~dB})$ whereas $\lambda=1200 \mathrm{~nm}$ doesn't obey the Bragg grating condition, and as a result, it propagates throughout the grating as shown in Figure $3 b$. 


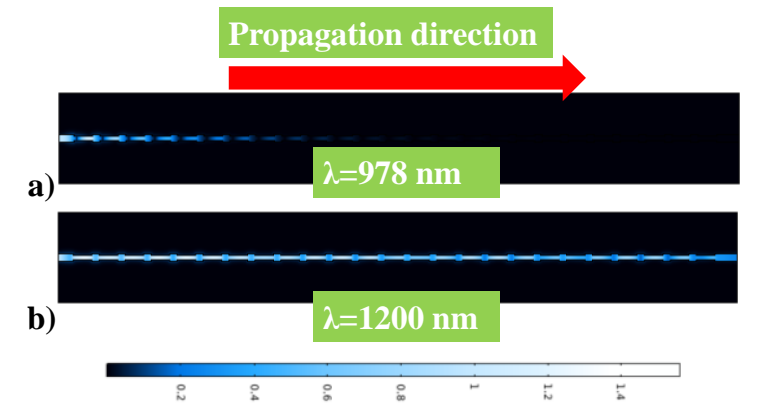

Fig. 3. E-field distribution in the Bragg grating structure at, a) $\lambda=978$ $\mathrm{nm}$, b) $\lambda=1200 \mathrm{~nm}$.

Moreover, $\lambda_{\text {Bragg }}$ can be tuned by varying the refractive index of the ambient medium, which results in variation of the effective refractive index of the propagating mode. For this analysis, the Bragg grating structure with parameters such as $L, s, w, d$ and $N$ fixed at $250 \mathrm{~nm}, 100$ $\mathrm{nm}, 100 \mathrm{~nm}, 20 \mathrm{~nm}$ and 25, respectively is used. From Fig. 4 it can be seen that $\lambda_{\text {Bragg }}=977 \mathrm{~nm}$ at $\mathrm{n}=1.0$ experiences a redshift to $1262 \mathrm{~nm}$ at $\mathrm{n}=1.3$ without changing the $E R$ of the filter. Additionally, the proposed Bragg grating structure can be used in refractive index sensing applications where the sensitivity $(S)$ can be calculated as [17-18]:

$$
S=\Delta \lambda_{\text {Bragg }} / \Delta \mathrm{n},
$$

where $\Delta \lambda_{\text {Bragg }}$ and $\Delta \mathrm{n}$ are the change in Bragg wavelength and refractive index, respectively. The proposed structure offers an $S=950 \mathrm{~nm} / \mathrm{RIU}$, which is higher than several previously proposed plasmonic sensors [19-20].

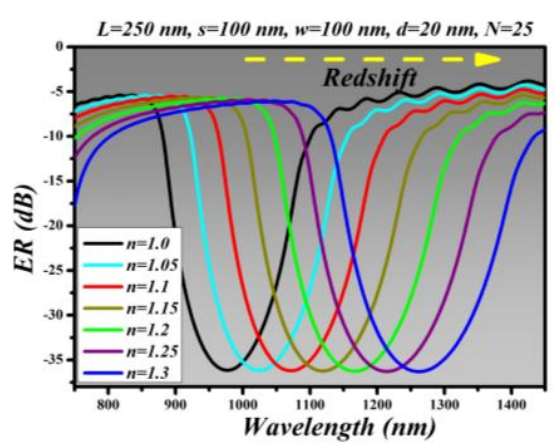

Fig. 4. The transmission spectrum of the Bragg grating structure versus the ambient refractive index.

In conclusion, we can say that a miniaturized design of a plasmonic Bragg grating structure is proposed which can be utilized in both filter and refractive index sensing applications. The spectral characteristics of the grating structure are numerically investigated by varying the period, strength of the grating and number of periods. Based on our results, for $N=25$, the maximum $E R$ and bandwidth of $36.2 \mathrm{~dB}$ and $173 \mathrm{~nm}$ are obtained with a filter footprint as small as $1 \times 8.75 \mu \mathrm{m}^{2}$, respectively. We believe that the $E R$ can be further increased by increasing the number of periods up to a limit where the plasmonic http://www.photonics.pl/PLP waveguide doesn't suffer from high propagation loss. Additionally, the Bragg grating structure is quite receptive to the refractive index of the medium. These characteristics allow the utilization of materials such as polymers in the metal-insulator-metal waveguide, which can be externally tuned or used for refractive index sensing applications. The sensitivity of the proposed Bragg grating structure can offer a sensitivity of 950 $\mathrm{nm} / \mathrm{RIU}$.

The author thanks the Samara National Research University and the Warsaw University of Technology for their support in completing this work.

\section{References}

[1] J. W. Field et al., 2019 Conference on Lasers and Electro-Optics Europe and European Quantum Electronics Conference (CLEO/Europe-EQEC), Munich, Germany (2019), doi: 10.1109/CLEOE-EQEC.2019.8871943.

[2] L. Cheng, S. Mao, Z. Li, Y. Han, H.Y. Fu, Micromachines 11, 666 (2020).

[3] J. Missinne, N.T. Beneitez, M-A. Mattelin, A. Lamberti, G. Luyckx, W.V. Paepegem, G.V. Steenberge, Sensors 18, 2717 (2018).

[4] M.A. Butt, S.N. Khonina, N.L. Kazanskiy, J. Modern Opt. 66, 1172 (2019)

[5] H. Qiu, J. Jiang, P. Yu, T. Dai, J. Yang, H. Yu, X. Jiang, Opt. Lett. 41, 2450 (2016).

[6] M.A. Butt, S.N. Khonina, N.L. Kazanskiy, Comp. Opt. 43, 1079 (2019).

[7] N.L. Kazanskiy, S.N. Khonina, M.A. Butt, Physica E 117, 113798 (2020).

[8] L. Lu et al., IEEE Photon. Technol. Lett. 22, 1765 (2012).

[9] R. Negahdari, E. Rafiee, F. Emami, J. Electromagn. Waves Appl. 32, 1925 (2018)

[10] M. Janfaza, M.A. Mansouri-Birjandi, Appl. Phys. B, 123, 262 (2017).

[11] C. Wu, G. Song, L. Yu, J.H. Xiao, J. Modern Opt. 60, 1217 (2013).

[12] J. Zhu, G. Wang, Results in Phys. 15, 102763 (2019).

[13] Y. Binfeng, H. Guohua, C. Yiping, Opt. Expr. 22, 28662 (2014).

[14] A.D. Simard, Y. Painchaud, S. Larochelle, Internat. Quantum Electr. Conference Lasers and Electro-Optics Europe, IEEE, Munich, Germany (2013).

[15] C. Klitis, G. Cantarella, M.J. Strain, M. Sorel, Opt. Lett. 42, 3040 (2017).

[16] J. Ctyroky et al., Opt. Expr. 26, 179 (2018).

[17] M.A. Butt, N.L. Kazanskiy, S.N. Khonina, Laser Phys. 30, 016202 (2020).

[18] M.A. Butt, N.L. Kazanskiy, S.N. Khonina, J. Modern Opt. 66, 1920 (2019)

[19] N.L. Kazanskiy, M.A. Butt, Photon. Lett. Poland 12, 1 (2020).

[20] Z. Guo, K. Wen, Q. Hu, W. Lai, J. Lin, Y. Fang, Sensors 18, 1348 (2018). 